\title{
Mobile-based traceability system for sustainable food supply networks
}

Kai-Yuan Lin ${ }^{1 *}$, David Chavalarias ${ }^{2,3 *}$, Maziyar Panahi ${ }^{2}$, Tsaiching Yeh ${ }^{4}$, Kazuhiro Takimoto $^{5}$, Masaru Mizoguchi ${ }^{1}$

Published in : Lin, K., Chavalarias, D., Panahi, M., Yeh, T., Takimoto, K., Mizoguchi, M., 2020. Mobile-based traceability system for sustainable food supply networks. Nature Food 1-7. https ://doi.org/10.1038/s43016-020-00163-y

\footnotetext{
${ }^{1}$ Laboratory of Agro-informatics, Graduate School of Agricultural and Life Sciences, The University of Tokyo, 7 Chome-3-1 Hongo, Bunkyo City, Tokyo 113-8654, Japan

${ }^{2}$ Complex Systems Institute of Paris Île-de-France, CNRS

${ }^{3}$ Centre d'Analyse et de Mathématiques Sociales, EHESS

${ }^{4}$ Independant Researcher, Tapei, Taiwan

${ }^{5}$ Independant Researcher, Tokyo, Japan

* Corresponding authors : Kai-Yuan Lin, E-mail : rgpstu15 at kmd.keio.ac dot jp; David Chavalarias, http ://chavalarias.org, E-mail : david.chavalarias at iscpif dot $\mathrm{fr}$
}

Abstract. Traceability is key to ensure food quality and safety from farm to fork, yet high implementation costs and the complexity of the food supply chain pose challenges to its operation. Here we propose a mobile-based bidirectional tracing system for food products that integrates graphic data and peer-to-peer $(P 2 P)$ architecture. Our system allows data synchronization to happen seamlessly between all connected nodes by default, as data are gathered through market transactions and all related product information is concatenated by scanning 2D barcodes on products. The system's decentralized and flexible structure favours bottom-up involvement by stakeholders and is applicable to various and dynamic food networks. Besides promoting resource efficiency and transparency of origin, production and distribution, the system can mesh surveillance and shed light on complex food networks, ultimately contributing to the advancement of food research.

\section{Introduction}

The expanding distance from farm to table, and the consequence of food scandals such as Bovine Spongiform Encephalopathy (BSE), Dioxin in chicken feed, Food-andMouth Disease (FMD) and issues such as the use of Genetically Modified (GM) crops, highlight the increasing importance and challenges to safety and quality along the food supply chain 1 .

Traceability can be used as an effective monitoring and management system with the potential to increase transparent information2, decreasing the incidence of food safety 
hazards, reducing the enormity and impact of such incidents by facilitating the identification of product(s) and/or batches affected, and to identify the problems and who is responsible 3,4 . Traceable information might also provide consumers with valuable information regarding the origin of food products or ingredients and might contribute to consumer beliefs regarding credence attributes, such as food safety ${ }^{5}$. Systems which allow traceability help to minimize the production and distribution of unsafe or poorquality products, thereby minimizing the potential for bad publicity, liability, and recalls. Further, increased transparency throughout the supply chain can reduce information asymmetry 1 .

With the trend in traceability, the world's two largest economies also require food traceability in regulations. EU directive 178/2002 requires mandatory traceability for all food and feed products sold within European Union countries ${ }^{6}$. In the US, the Bioterrorism Act of 2002 mentioned that the person who manufactures, processes, packs, transports, distributes, receives, holds, or imports food has the responsibility to establish and maintain records ${ }^{1}$.

The current major challenges in traceability are compounded by the cost of implementation such as system construction, manpower, time, extra workload, and complexity in the supply chain. In general, only large-scale production or high-priced products have a high ability to control the supply chain and traceability in a systematic and rigorous way, such as the Marine Stewardship Council (MSC) label, Nordic fish products7, and Japanese wagyu ${ }^{8}$. However, not all food companies have sufficient economic value or scale to invest in a traceability system, especially small firms may experience cost disadvantages 9 . Furthermore, owing to the lack of uniformity in the traceability systems used, coordination and allocation of costs and the benefits among various actors across the chain may also be affected ${ }^{10,11,12}$. Therefore, a well-designed traceability mechanism is necessary for multi-scale producers as a bottom-up resolution of the health-dietenvironment trilemma. The development of legal systems compatible with the new regulatory framework is needed in policymaking, and for prompt and effective implementation. Extensive modalities of information and communication technologies (ICT) are realizable in the era of the Fourth Industrial Revolution, in close coordination with global sustainable development goals ${ }^{15}$. Nowadays, data is the essential ingredient in food production to connect participants across the food supply chain to mitigate information asymmetry. The $\mathrm{P} 2 \mathrm{P}$ technology is a powerful tool and emerging topic in recent food traceability research ${ }^{16,17}$. P2P systems inherently have high scalability, robustness, and fault tolerance because there is no centralized server and the network self-organizes itself ${ }^{18}$. It has the characteristics of high throughput, low latency, powerful query functionality, decentralized control, immutable data storage19, and built-in asset support. All of which has the high potential and usability to tackle the complexity of traceability within diverse supply chain. Blockchain is known as a common technology base on P2P network. Although it has some benefits and applications in finance, cryptocurrency and some agri- 
food supply chains, it still faces some limitations when it comes to implementation ${ }^{20,21}$, such as accessibility for resource-poor enterprises ${ }^{22}$, the high energy consuming due consensus mechanism ${ }^{20}$, inefficient storage and scalability obstacles ${ }^{23,24}$, which remain problem in adopting traceability.

The aim of this research is to improve the popularization of the traceability system and solidify the food supply network. We designed a mobile-based P2P traceability system which discusses a set of data-driven gathering, exchange, and storage systems with decentralized, user-friendly operational frameworks that can be applied in different production and distribution modes. By allowing the "precise and comprehensive traceability" to be implemented more broadly and easily, it supports the management of multi-scale and diversified food producers that are relatively underprivileged but more sustainable, helping to balance the supply chain information asymmetry.

\section{A. Conventional system}
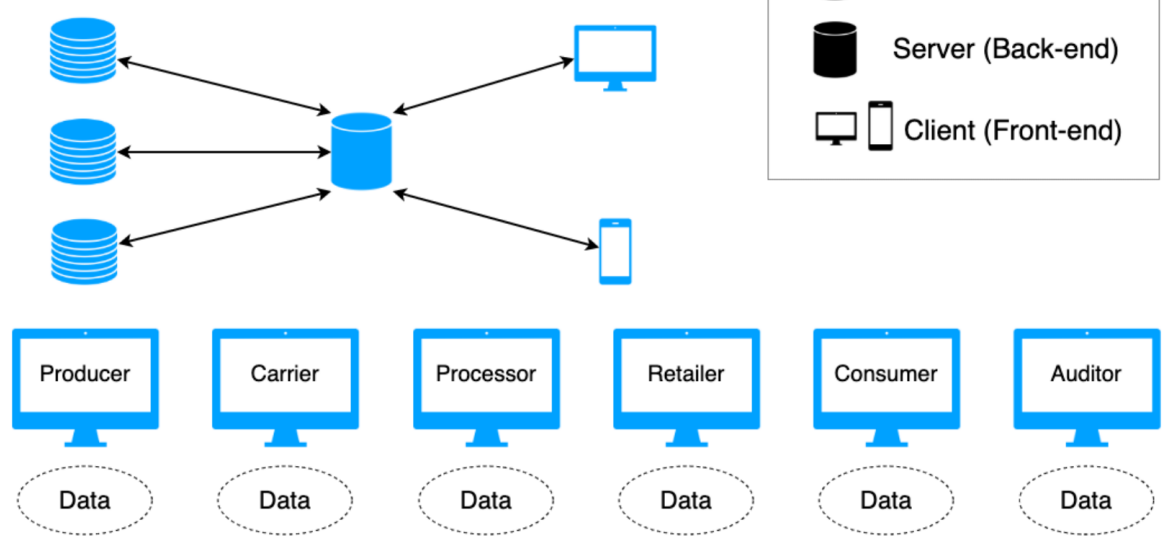

\section{B. Proposed system}

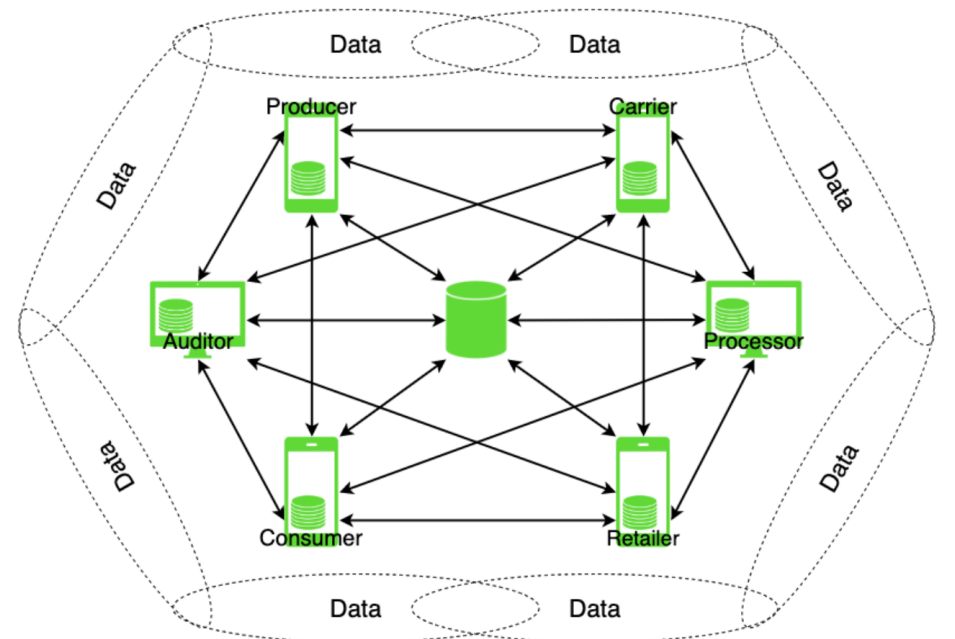

FIGURE 1 - Information from linear to mesh structure. The stacked-pie chart represents the database server. They cylinder-shaped chart represents the back-end server. Computer and mobile icons represent the client front-end. (a) Conventional system : the server is centralized and each unit holds its own information that is not connected. (b) Proposed mesh structure : Personal devices act as the database server, gives the information equality. Moreover, all information between the supply chain is interconnected, and each unit can easily trace back to the upper provider. 


\section{System Design : Mobile-based P2P traceability}

\subsection{Design concept : mesh structure in information}

Conventionally, traceability data is stored independently by individuals within the supply chain, it is extremely time and labor consuming for end-to-end data gathering (Figure $1 \mathrm{~A}$ ).

We designed the system for transparency and created the mesh structure of communication for the food supply network, which enables individuals to coordinate and interlock supply chains to produce or service collectively ${ }^{25}$ (Figure 1 B). It is more customer driven and works in a less linear operation than conventional supply chain management systems. Given that the role of the different stakeholders in a supply chain has become increasingly blurred in recent times, the mesh structure reveals the bidirectional communications and interactions of individuals, which restores the comprehensibility of the whole process. Moreover, by reducing the information asymmetry, the mesh structure enables the supply chain to choose their suppliers of sources fairly and increase the rights for the consumer. In this paper, we propose the integration of information concatenation with mobile-based $\mathrm{P} 2 \mathrm{P}$ network coupled to a graph database, for high versatility and a user-friendly approach that implements an ideally mesh structure over stakeholders' communication and information. This low-cost self-management tool for multi-scale holders is expected to further reinforce the food supply network with mesh surveillance and information symmetry while lowering entry barriers to the precise and complete traceability.

\subsection{Graph database and 2D barcode for information concatenation}

The building blocks of the mesh structure for food supply chain is a process of information concatenation that describes each newly generated piece of information in the database and inherits the full line of (or lineage of) associated upstream information. We propose a $2 \mathrm{D}$ barcode as a common language for product identification in the system, to both carry and transfer the information as well as act as the media to access the information. It is commonly used in tracking and tracing initiatives because they are flexible in size, offer high fault tolerance and have fast readability, which can be read by any mobile device $^{26}$.

Not only is basic traceability information recorded, but users can also add any additional information to the database, which keeps the flexibility in addressing the diversity of products, production scales, and regulations. We chose graph database, which can easily trace and track the end to end information of a specific point ${ }^{27}$. It offers infinite scalability which plays a crucial role in our proposed architecture on top of a decentralized network. The graph database offers operations which are in harmony with the data flow in our architecture. In our preliminary studies, we concluded that graph databases will be more performant due to the required data-flow in our system, which makes them perfect 
candidates to be used in smaller devices such as phones, on a slow network connection, and a real-time architecture. (More technological explanation in supplementary material $1 \&$ supplementary fig. S1 \& S2).

In this system, consumers and producers are not specifically distinguished ${ }^{28}$, as long as the registered ID holders receive the product from others, they are interlinked. Through the mechanism of information concatenation, the transaction process of the product generates the new route of its barcode with the responsible IDs, which also allows the upstream information to be inherited to the next barcode. The traceability in the supply chain is therefore achieved without additional constraints, information is intuitively collected, which also reduces the workload in data collection, and the chain continues as long as the product keeps transacting to the next party.

\subsection{Information decentralization with Mobile-based P2P}

Information about products stored into $2 \mathrm{D}$ barcode should be easily accessible throughout the entire mesh structure. We propose to rely on a P2P technology that combines high availability with high robustness ${ }^{29}$. The decentralized nature of P2P networks allows actors in a supply chain to share surplus computing and storage resources on their personal devices with the entire network as peers ${ }^{30}$. The structure becomes firmer when peers increase. Mobile-based services allow any personal device to download the service from the back-end server to become a peer, which reduces the entry barrier and increases the versatility for multi-scale production. The mobile-based P2P has a lightweight design in read and input, allowing low storage capacity devices to utilize temporal storage before the data wildly spreads, and offline usage when it faces a weak connection. Even consumers can join and contribute to the P2P network, which extends the network and reduces the cost for managing the servers, and can be started among any production community. Servers (super-peer) can be introduced to enhance the functionality of the system and its robustness. (in the middle of fig. 1 B). The access frequency of old data decreases, and the characteristic of short consuming dates in food supply chain does not require the long term keeping of data. Therefore, an archiving strategy can be implemented where the older finished cases can be moved to super-peers after a chosen period of TTL (Time -to-live). Archiving can compress the data properly based on the access frequency, or keep only the hash of data which prevents tampering.

This technologic approach will enable end-to-end traceability by bringing a common technological language to the food chain, while both allowing consumers to access the story of their food products on their phones through their label and shortening the distance with producers ${ }^{31}$. P2P increases robustness and security because it removes the single point of failure and control that can be inherent in a client-server based system ${ }^{32}$. Even the system manager or super-peer is only acting as a high-performance node among others (in terms of computational resource and storage). Once the information is uploaded, it belongs to the whole network instead of a specific party, which is essential in the 
complete traceability process. Each piece of information provides critical data that may potentially reveal food safety issues with the product concerned ${ }^{31}$.

\section{System operation and user scenario}

The system is designed to allow food industries to coordinate their value chains via information technology by integrating the business processes of customers, suppliers, and trading partners among different companies in the industry. The system is flexible and versatile enough to assist those multi-scale producers who account for a large number of markets and the diversity of their products and production models ${ }^{3}$. Once the 2D barcode is generated, it will be encrypted and uploaded to the database and duplicated in the P2P network. Transaction of the product and regeneration based on this 2D barcode relies on the consensus between two parties at various locations in the reality, which creates many chains in the database. Further, one product (barcode) can be traded to different places, which means one barcode can have multiple transactions based on multiple consensuses. It is extremely hard to tamper with or fake the chain, due to all divergence or convergence branches of this chain which must be taken in to concern. It shows that chains are loose coupling, which indicates the aggregation of consensus. This system functions in three parts (Figure 2) : barcode generation, transaction, and access right. (See more explanation about mobile interface and function of the proposed system in supplementary fig. S3)

\subsection{ID registration \& product barcode generation}

Clear record of responsible party in traceability among the supply chain contributes to the more precise recall and management ${ }^{33}$, therefore, the ID registration in the system is mandatory to join the supply chain. As fig. 2 (a-1), every time the product is physically or chemically changed, the responsible ID holder should generate a new barcode. If the product has associated ingredients or components that the producer is utilizing or adding to modify the product, the producer is required to scan all of their barcodes prior to generating the new barcode. The scanning action realizes the information concatenation to ensure the accuracy of traceability. Later, input the product information or associated documents to generate the new barcode. Part of the mandatory product information which is required by law is embedded into the barcode for people to gain in the offline environment.

\subsection{Transaction}

Refer to the Food Traceability Guidance provided by FAO34, the system will always record the responsible ID, product name, time and location for every transaction. This prevents acts of dishonesty or misuse of the barcode for not only producers but also allows related stakeholders among supply chains to inspect whether the new route is 
valid. The traceability continues via transaction (Figure 2 a-2), and we have designed a two-factor authentication process for verification to avoid misuse and specify the responsibility (Figure 3 step 2-3). The same action can be taken in a relay scenario to record the second transaction of products. Our offline first capabilities mean that if connectivity is lost to other nodes due to a network error or no availability, the application will store all changes locally and automatically synchronize as soon there is a connection. Additionally, memo or modification can be added during and after the transaction, however, the edited record will remain.

\subsection{Access right and Tree map}

Information will be divided into product and transaction information, which allows different access rights (Figure 2 a-3). Anyone can read the product information and the concatenated information via scanning the barcode, and easily trace the tree map, however, with the permission function in the graph database, only the barcode producer can track the forward traceability. (See different user scenario in Figure 2 (b)) Further, the product from the same batch might be transacted to various places by different people, these transactions are partially commercially confidential, therefore can only be viewed by associated ID. Traceability systems help firms isolate the source and extent of safety or quality control problems. The more precise the tracing system, the faster a producer can identify and resolve food safety or quality problems ${ }^{35}$. Our system enables the producers to track forward and understand what their products are used for, and the consumers can trace back upstream to understand the origin of the source. This mechanism completes the tree map of traceability, which accelerates the time for defining problem points and response times for recalls when any incident occurs. In addition, other relative supply chains can also be notified faster if they have been affected. As long as there is a connection between products, the scope of traceability will not be limited.

\section{Discussion}

\subsection{Comparison with Blockchain technology solution}

The latest hype in the field of food traceability associated with information and communication technology is Blockchain. We completed extensive research before ruling out Blockchain technology as our primary choice for decentralization, figuring out some

of the drawbacks and limitations ${ }^{24,32,37,38}$ when it comes to implementation and requirements of the issue. Some notable features in our architecture compared to Blockchain technology are shown in Table 1. 


\section{(a) System function \& operation}

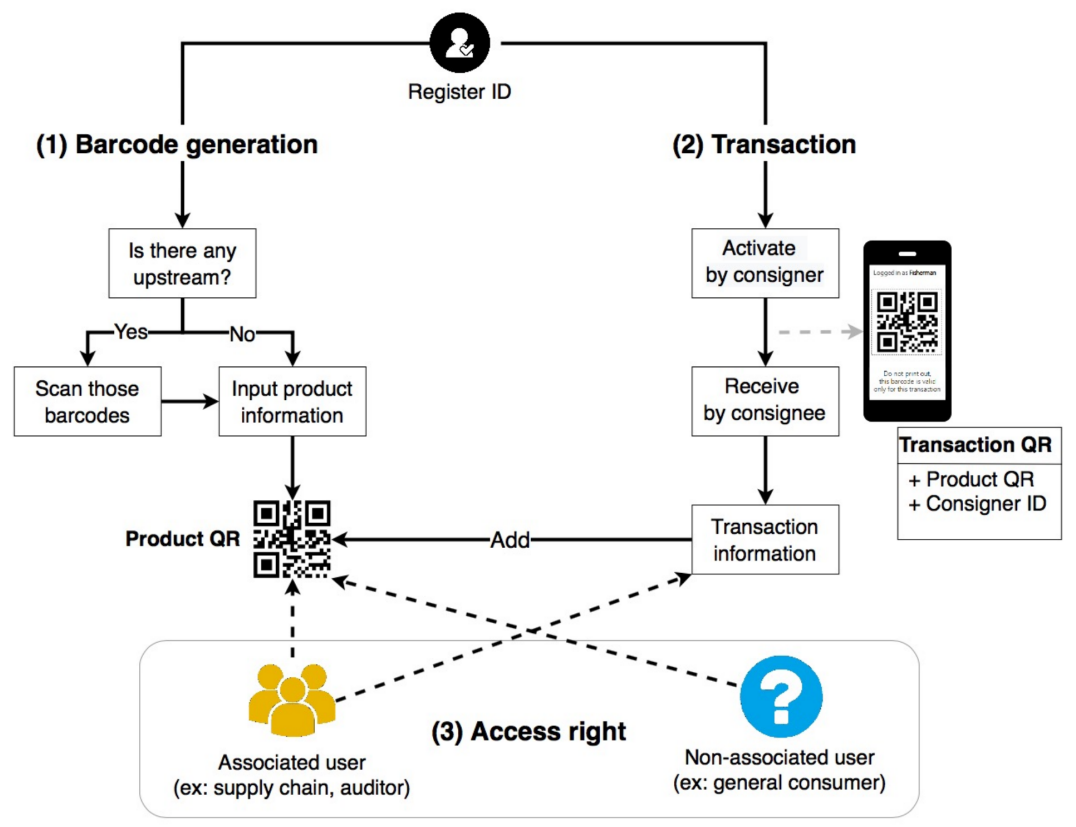

\section{(b) User scenario: tracing and tracking}

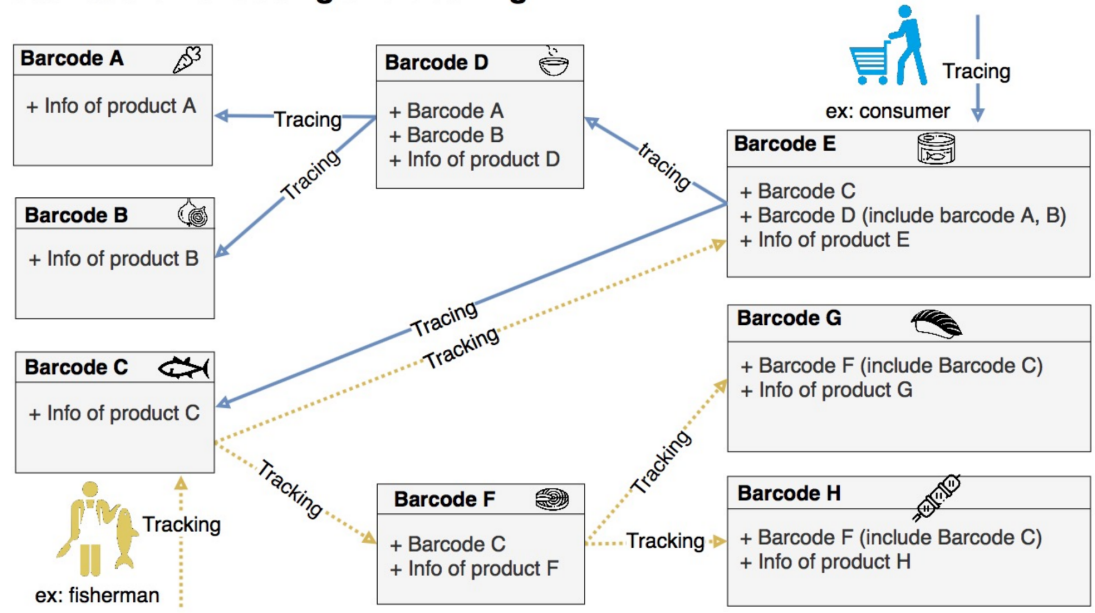

FIGURE 2 - System function and scenario. (a) System function performs in three parts : barcode generation, transaction and access right. (1) Barcode generation : Receive the upstream product information via transaction, and input to the new generated barcode with product information. (2) Transaction : Firstly, in the "Activate step", consigner scans the barcode of the product, and their interface will appear with the temporal 2D barcode (function and interface please see supplementary fig. S3), which represents the action that is taken by the consigner. The consignee then scans this temporal 2D barcode and completes the transaction, which is the "Receive step". The same action can be taken in a relay scenario to record the second transaction of products. The process above allows the product information to be inherited to the associated product later. (3) Access right : Information divided into product and transaction information, which allows different access rights based on associated and non-associated ID. (b) Tracing \& tracking : The boxes show the associated upstream information in the barcode. ID holders can view the supply chain information related to themselves. Blue lines show the end-to-end tracing scenario and contents for consumers, when they scan barcode $\mathrm{E}$, the information of barcode $\mathrm{C}$ and $\mathrm{D}$ will be unfolded, and further reach barcode $\mathrm{A}$ and $\mathrm{B}$ from barcode D. Yellow lines show the end-to-end tracking scenario and contents for producers. They can track barcode F, E, G and H by scanning barcode C, but the access right does not allow s/he to read the non-associated information such as barcode A, B and D. 


\section{(a) System function \& operation}

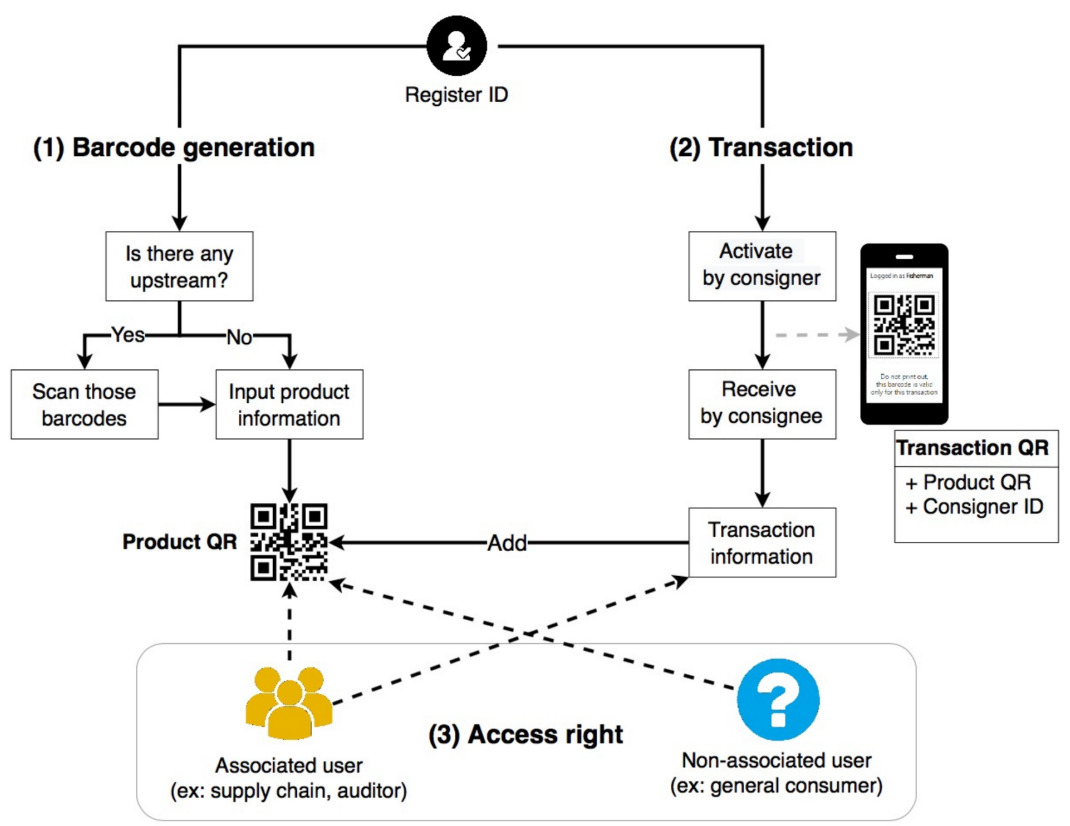

\section{(b) User scenario: tracing and tracking}

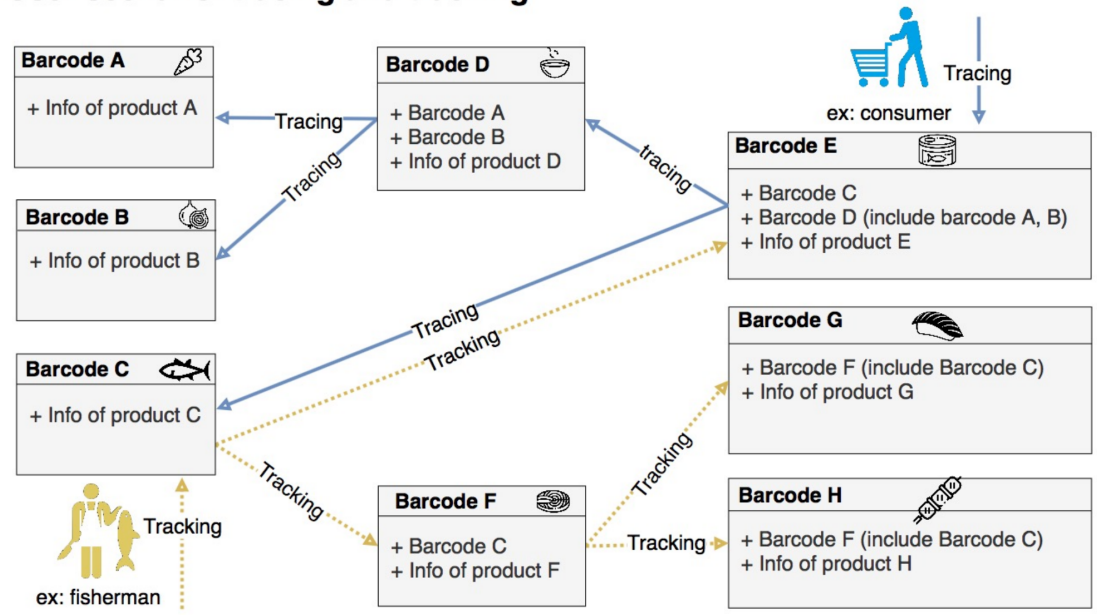

FIGURE 3 - System function and scenario. Scenario illustration of system operation and the content in the database. User action in the food supply chain and the corresponded records generated in the database. Step1, producer1 generates the new barcode, database record its ID. Step 2, for transaction, the consigner scans the barcode of the product and a temporal 2D barcode appears for transaction, which represents this action taken by the consigner. Step 3, the consignee then scans this temporal 2D barcode and completes the transaction, which is the receive process. Step 5-6, same as step 2-3. Step 7, every time when the product is repacked or reproduced, it is mandatory to generate a new barcode. Item 2 included the information of item 1 for information concatenation. Step 8-9 and Step 11-12, same as Step 2-3. Step 13, in the relay scenario, the product does not have any physical or chemical change, therefore, it only adds the transaction record to the original barcode instead of generating the new one. 


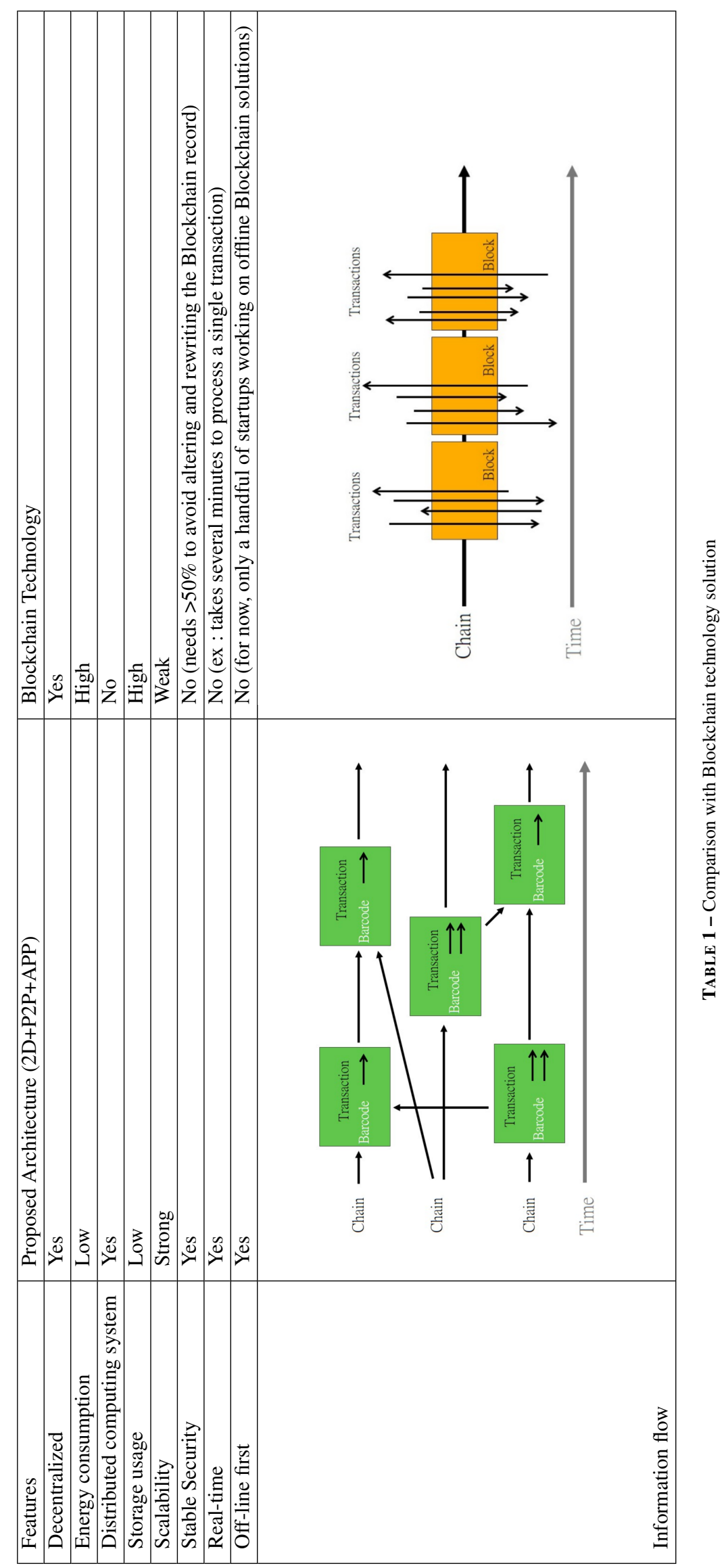


1. High energy consumption ${ }^{39,40}$ : Non-associated data will be merged into a block at a certain time based on the designed algorithms for verification. This consensus mechanism such as PoW or PoS will converge data to a single chain. The competing miners in Blockchain technology use a disproportionate amount of electricity to create the next block. Our architecture relies entirely on mobile phones because of their availability among food producers, delivery trucks, distributors, and endusers. We rely on the BYOP (Bring Your Own Phone) approach for the feasibility of the final implementation from both the financial perspective and flattening the learning curve for all the involved parties. Although modern mobile phones can be used throughout the day dependent on battery life, our application will not be the only item on those devices. The users still need to be able to make calls, use geolocalized apps for direction, messaging apps, etc. The high consumption of energy in Blockchain makes the choice of mobile phones impossible if they are not continually plugged into a charger throughout entire period of use. On the other hand, our system relies on the actual transaction between two parties, the consumption for consensus mechanism is based on the energy flow from the real world.

2. Blockchain is not actually a distributed computing system : The assumption that Blockchain is some sort of a distributed computing system with nodes across different geo locations is incorrect. In reality, all of the nodes which maintain a Blockchain do exactly the same thing while distributed, it is not a distributed computing system that will benefit all. Our proposed architecture is not only decentralized and distributed, but also has the ability to operate in parallel. For instance, the operations related to the database such as insert, updates, and queries are executed in parallel which helps to turn all the phones into a distributed computing system where the devices can operate in parallel while executing different operations independently.

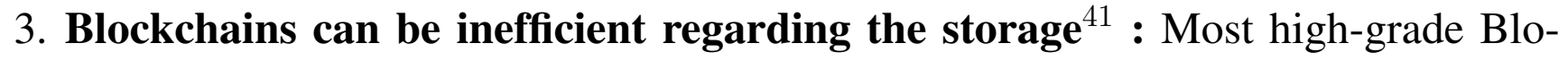
ckchain network clients will store the entire transaction's history. For example, in the case of Bitcoin, the records can exceed over 100GB which is way beyond the average storage capacity of a smartphone today. To store Blockchain data, that data has to be downloaded and the verification process must be completed. Even with recent advances in portable storage devices, the Blockchain technology is not a viable choice for a long-term system depending entirely on mobile devices.

4. Blockchain remains weak when it comes to scalability ${ }^{23,42}$. Blockchains are not scalable compared to our proposed architecture. In other words, the more people or nodes that join the Blockchain network, the slower the network becomes. Although there has been an increasing number of solutions to overcome this, such as only store and access information on a Blockchain network, it is still not well equipped for real-world applications. It has been cited by many researchers and engineers that Blockchain technology requires significant improvements in speed and scalability 
before it can be adopted into our day-to-day life ${ }^{43,44}$. The distributed computing system in our architecture allows easy scalability through the entire network and has notable advantages over Blockchain technology.

Moreover, with concern to the feasibility of price, reliability in the shipping industries, and the learning curve forced upon the involved parties, our system is superior. The proposed architecture only requires a printer and a phone with internet and camera which makes it easy to be implemented in multiple and diversified scenarios.

\subsection{Extension and enhancement in traceability}

1. Flexibility in TRU. A Traceable Resource Unit (TRU) is defined as a homogeneous collection of one resource class that is used/consumed/produced/released by a primitive activity in a finite, non-zero quantity of that resource class ${ }^{36}$. Products from small producers demand differentiation and have a higher chance that the suppliers and the ingredients will change, thus the quality of each batch may be different. In addition, generation of the barcode based on the batch is highly recommended for precise traceability, which means consignees will not have the newest barcodes if they did not receive the product from the consigner. This mechanism prevents food fraud and adulteration. With the high flexibility to input data in our system, it seems easy to arrange different TRUs according to different needs for batch control and management, overcoming the inefficiency of production. This information can also be quickly displayed in the system, showing different values of each batch of products.

2. Forward traceability and proof of origin With the information concatenation nature in our system, associated information of the supply chain is linked together. Thus, people do not need to rely on specific organizations or third-party certification to prove the origin, which reduces the cost for producer and increases the consumer trust. Forward traceability allows producers to track the trade path of their products and have more power in quantity control to manage and protect their brand. Moreover, if the total output of the region has been recorded in the system, the production volume, after deducting the consumption during processing, should be equal to the inventory and sales volume in the final consumer market, which can be used to prevent the adulteration. Our system records the responsible parties for products among the entire supply chain. Further, we believe that enabling the forward traceability from the source improves the food safety and quality. Traceability can be seen as an effective tool for supply chain to improve the quality of raw materials ${ }^{36}$. Clear origin of ingredients reactivates and supports the sustainable trends in community-based production and could help shorten the transportation distance. Moreover, consumers often prefer products from a specific geographical origin or a particular brand. 
3. Extend the data range and other application. We provide an extensive underlying system framework that allows different interconnected traceability applications to be designed and developed, which can be combined with other systems as plug-in information for more comprehensive food supply-chain records. Apart from the direct impact on the food industry and its internal organization, we expect that the proposed systems will have a significant impact on consumers behavior through the possibility of product information enrichment in buying situations. (See more explanation in supplementary material 2 \& supplementary fig. S4). Recently consumer concerns have extended to non-food producers which are also associated with the supply chain. For example, consumers are concerned with the processing and production that attributes organic, natural, or genetically modified origins, as well as environmental and sustainable issues such as the use of energy, resources, and the emission of Green House Gases (GHG), or packaging and recycling. Another useful application would be in social issues such as consumer justice, animal and human welfare, and agricultural operations ${ }^{1,35,45,46}$. In terms of producer management, our system could combine the information required for the trade, such as non-IUU data of fishing, agricultural operation management records, and inspection reports, or it could combine with the point-of-sale (POS) systems to manage and analyze sales data. Also, by combining with sensors in refrigeration equipment, and recording and reporting real-time monitoring data, producers can have better control and management of the quality of products and supply chain. These issues cannot be addressed with traditional food and product labeling but can easily be addressed with future extensions of our P2P system. Although it is not the place here to expose such system details, we would like to give the broader picture that could be built upon with our traceability system.

\section{Conclusion}

The mobile-based P2P traceability system offers offline-first, and a real-time application using 2D barcodes over a decentralized P2P network that benefits from end-to-end encryption and tamper-proof solution. It is expected to improve the information disconnection in the conventional system and provide higher resilience with scalability, while facilitating the food supply chain to manage its products and enhance its added value. In response to the increasing demand for supply chain transparency, the proposed system is expected to become an efficient tool in traceability for both consumers and producers. The information on products is often scarce, expensive, or even sometimes impossible to obtain. This results in imperfect information for consumers, a situation known to lead to significant market failures ${ }^{29}$. In the proposed system, we integrate security as well as the equality of information by decentralization, higher efficiency, and uninterrupted 
traceability by information concatenation. By being both 2D barcode and mobile-based, the system can be used more widely with flexibility. Through the bottom-up strength it is anticipated to increase the transparency and information accuracy in diverse food production. We provide a system for multi-scale holders to coordinate their supply network via information technology to produce a product or service for a market collectively by synchronizing the business processes of customers, suppliers, and trading partners among different parties in an industry. Clear origin of ingredients will help reactivate, support, and help to sustain the trend in community-based production, as well as shortening transportation distances. Ensuring ultimate traceability means giving people back their rights to product information.

\section{Acknowledgments}

We would like to express the special thanks of gratitude to Collette Kuntz from Swedish Medical Center, and Chieh-Wen Lo from The University of Tokyo, your kind advice and help are indispensable to this research. We also thanks to Masatoshi Funabashi, your philosophy inspires us for the studies.

\section{Author Contributions}

- Kaiyuan Lin and Tsaiching Yeh develop the study concept and design the system,

- Maziyar Panahi and Kazuhiro Takimoto contribute to the system architecture

- David Chavalarias contributes to the future application (LES).

\section{Competing Interests statement}

Authors declare no competing interests.

\section{References}

1. Aung, M. and Chang, Y. Traceability in a food supply chain : Safety and quality perspectives. Food Control 39, 172-184 (2014).

2. Beulens, A., Broens, D., Folstar, P. and Hofstede, G. Food safety and transparency in food chains and networks Relationships and challenges. Food Control 16(6), 481-486 (2005).

3. Opara, L. U. Traceability in agriculture and food supply chain : a review of basic concepts, technological implications, and future prospects. Food, Agriculture \& Environment 1(1), 101-106 (2003).

4. Wilson, T. P., \& Clarke, W. R. Insights from industry food safety and traceability in the agricultural supply chain : using the Internet to deliver traceability. Supply Chain Management 3(3), 127-133 (1998).

5. Kher, S., Frewer, L., Jonge, J., Wentholt, M., Davies, O., Luijckx, N. and Cnossen, H. Experts ' perspectives on the implementation of traceability in Europe. British Food Journal 112(3), 261-274 (2010).

6. Folinas, D., Manikas, I., \& Manos, B. Traceability data management for food chains. British Food Journal 108(8), 622-633 (2006).

7. Randrup, M., Storøy, J., Lievonen, S., Margeirsson, S., Árnason, S., Ólavsstovu, D., Møller, S. and Frederiksen, M. Simulated recalls of fish products in five Nordic countries. Food Control 19(11), 1064-1069 (2008).

8. Clemens, Roxanne L. B. Meat Traceability in Japan, Iowa Ag Review 9 (4), Article 2, (2015). Available at : http ://lib.dr.iastate.edu/iowaagreview/vol 
9. Sodano, V. and Verneau, F. Traceability and food safety : public choice and private incentives, quality assurance, risk management and environmental control in agriculture and food supply networks. Proceedings of the 82nd Seminar of the European Association of Agricultural Economists (EAAE), Bonn, Germany, 14-16 (2004).

10. Disney, W.T., Green, J. W., Forsythe, K.W., Wiemers, J.F., and Weber, S. Benefit-Cost Analysis of Animal Identification for Disease Prevention and Control. Rev. Sci. et Techique de l'Office Internacional des Epizooties 20, 385-405 (2001).

11. Petit, R.G. Traceability in the Food Animal Industry and Supermarket Chains. Rev. Sci. et Techique de l'Office Internacional des Epizooties 20, 584-597 (2001).

12. Vitiello, D. J. and A. M Thaler. Animal Identification : Links to Food Safety. Revue Scientifique et Techique de l'Office Internacional des Epizooties 20, 598-604 (2001).

13. Herrero, M., Thornton, P., Power, B., Bogard, J., Remans, R., Fritz, S., Gerber, J., Nelson, G., See, L., Waha, K., Watson, R., West, P., Samberg, L., van de Steeg, J., Stephenson, E., van Wijk, M. and Havlík, P. Farming and the geography of nutrient production for human use : a transdisciplinary analysis. The Lancet Planetary Health 1(1), 33-42 (2017).

14. Pomeroy, R.S. and N.L. Andrew. Small-Scale Fisheries Management : Frameworks and Approaches for the Developing World. CABI (formerly CAB International), Wallingford, UK and Cambridge, MA, USA, 247 (2011).

15. Funabashi, M. Human augmentation of ecosystems : objectives for food production and science by 2045. npj Sci Food 2, 16 (2018).

16. Bechini, A., Cimino, M., Marcelloni, F. and Tomasi, A. Patterns and technologies for enabling supply chain traceability through collaborative e-business. Information and Software Technology 50(4), 342-359 (2008).

17. Mao, D., Wang, F., Hao, Z. and Li, H. Credit Evaluation System Based on Blockchain for Multiple Stakeholders in the Food Supply Chain. International Journal of Environmental Research and Public Health 15(8), 1627 (2018).

18. Singh, K. and Schulzrinne, H. Peer-to-peer internet telephony using SIP. Proceedings of the international workshop on Network and operating systems support for digital audio and video, 63-68 (2005).

19. Zheng, X., Zhu, Y., and Si, X. A Survey on Challenges and Progresses in Blockchain Technologies : A Performance and Security Perspective. Applied Sciences 9(22), 4731 (2019).

20. Kamilaris, A., Fonts, A. and Prenafeta-Boldú, F.X. The rise of blockchain technology in agriculture and food supply chains. Trends in Food Science \& Technology 91, 640-652 (2019).

21. Feng, H., Wang, X., Duan, Y., Zhang, J. and Zhang, X. Applying blockchain technology to improve agri-food traceability : A review of development methods, benefits and challenges. Journal of Cleaner Production, 121031 (2020).

22. Ge, L., Brewster, C., Spek, J., Smeenk, A., Top, J., van Diepen, F., Klaase, B., Graumans, C. and de Wildt, M.D.R. Blockchain for agriculture and food : Findings from the pilot study. Wageningen Economic Research, 2017-112 (2017).

23. Eyal, I., Gencer, A. E., Sirer, E. G., \& Van Renesse, R. Bitcoin-ng : A scalable blockchain protocol. Proceedings of the 13th USENIX symposium on networked systems design and implementation (NSDI). Santa clara, CA, USA, 45-59, (2016).

24. Pearson, S., May, D., Leontidis, G., Swainson, M., Brewer, S., Bidaut, L., et al. Are distributed ledger technologies the panacea for food traceability? Global Food Security 20, 145-149, (2019).

25. Skinner, Chris. ValueWeb : How fintech firms are using bitcoin blockchain and mobile technologies to create the Internet of value. Marshall Cavendish International Asia Pte Ltd, (2016).

26. Gao, J.Z., Prakash, L. and Jagatesan, R. Understanding 2d-barcode technology and applications in m-commerce-design and implementation of a $2 \mathrm{~d}$ barcode processing solution. IEEE In 31st Annual International Computer Software and Applications Conference (COMPSAC 2007) 2, 49-56, (2007).

27. Dubey, A., Hill, G.D., Escriva, R. and Sirer, E.G. Weaver : a high-performance, transactional graph database based on refinable timestamps. arXiv preprint arXiv :1509.08443, (2015).

28. Macias, T. Working Toward a Just, Equitable, and Local Food System : The Social Impact of Community-Based Agriculture. Social Science Quarterly 89(5), 1086-1101 (2008).

29. Fan, B., Andersen, D.G., Kaminsky, M. and Papagiannaki, K. Balancing throughput, robustness, and in-order delivery in P2P VoD. In Proceedings of the 6th International COnference , 1-12, (2010).

30. Lua, E. K., Crowcroft, J., Pias, M., Sharma, R., Lim, S. A survey and comparison of peer-to-peer overlay network schemes. IEEE Communications Surveys \& Tutorials 7(1-4), 72-93 (2005).

31. Litke, A., Anagnostopoulos, D. and Varvarigou, T. Blockchains for supply chain management : Architectural elements and challenges towards a global scale deployment. Logistics 3(1), 5, (2019).

32. Galvez, J., Mejuto, J. and Simal-Gandara, J. Future challenges on the use of blockchain for food traceability analysis. TrAC Trends in Analytical Chemistry 107, 222-232 (2018).

33. Bosona, T. and Gebresenbet, G. Food traceability as an integral part of logistics management in food and agricultural supply chain. Food Control 33(1), 32-48 (2013).

34. FAO. Food Traceability Guidance. Retrieved from http ://www.fao.org/3/a-i7665e.pdf (Accessed 24.02.2020) (2017). 
35. Golan, E., Krissoff, B., Kuchler, F., Calvin, L., Nelson, K., \& Price, G. Traceability in the U.S. food supply : Economic theory and industrial studies. Agricultural Economic Report, 830 (2004).

36. Galvão, J. A., Margeirsson, S., Garate, C., Viðarsson, J. R., \& Oetterer, M. Traceability system in cod fishing. Food Control 21, 1360-1366 (2010).

37. Tian, F. A supply chain traceability system for food safety based on HACCP, blockchain \& Internet of things. In 2017 International conference on service systems and service management. IEEE, 1-6, (2017).

38. Caro, M.P., Ali, M.S., Vecchio, M. and Giaffreda, R. Blockchain-based traceability in Agri-Food supply chain management : A practical implementation. In 2018 IoT Vertical and Topical Summit on Agriculture-Tuscany (IOT Tuscany). IEEE, 1-4, (2018).

39. Krause, M. J., \& Tolaymat, T. Quantification of energy and carbon costs for mining cryptocurrencies. Nature Sustainability 1, 711-718, (2018).

40. Truby, J. Decarbonizing Bitcoin : Law and policy choices for reducing the energy consumption of Blockchain technologies and digital currencies. Energy research \& social science 44, 399-410, (2018).

41. Tang, Y.R., Xing, Z., Xu, C., Chen, J. and Xu, J. Lightweight blockchain logging for data-intensive applications. In International Conference on Financial Cryptography and Data Security, 308-324, (2018). Springer, Berlin, Heidelberg.

42. Tribis, Y., El Bouchti, A., \& Bouayad, H. Supply chain management based on blockchain : A systematic mapping study. MATEC web of conferences (EDP sciences) 200, (2018).

43. Vukolić, M. The quest for scalable blockchain fabric : Proof-of-work vs. BFT replication. In International workshop on open problems in network security, 112-125, (2015). Springer, Cham.

44. Xu, X., Pautasso, C., Zhu, L., Gramoli, V., Ponomarev, A., Tran, A.B. and Chen, S. The blockchain as a software connector. In 2016 13th Working IEEE/IFIP Conference on Software Architecture (WICSA), 182-191, (2016). IEEE.

45. Caswell, J.A. How labeling of safety and process attributes affects markets for food. Agricultural and Resource Economics Review 27, 151-158 (1998).

46. Nelson, P. Information and consumer behavior. Journal of Political Economy 78, 311-329 (1970). 